\title{
Construction of Bordetella pertussis strains with enhanced production of genetically-inactivated Pertussis Toxin and Pertactin by unmarked allelic exchange
}

Wasin Buasri ${ }^{1}$, Attawut Impoolsup ${ }^{1}$, Chuenchit Boonchird', Anocha Luengchaichawange ${ }^{2}$, Pannipa Prompiboon², Jean Petre $2^{2^{*}}$ and Watanalai Panbangred ${ }^{1,3^{*}}$

\begin{abstract}
Background: Acellular Pertussis vaccines against whooping cough caused by Bordetella pertussis present a muchimproved safety profile compared to the original vaccine of killed whole cells. The principal antigen of acellular Pertussis vaccine, Pertussis Toxin (PT), must be chemically inactivated to obtain the corresponding toxoid (PTd). This process, however, results in extensive denaturation of the antigen. The development of acellular Pertussis vaccines containing PTd or recombinant PT (rPT) with inactivated S1, Filamentous Hemagglutinin (FHA), and Pertactin (PRN) has shown that the yield of PRN was limiting, whereas FHA was overproduced. To improve antigen yields and process economics, we have constructed strains of Bordetella pertussis that produce enhanced levels of both rPT and PRN.
\end{abstract}

Results: Three recombinant strains of Bordetella pertussis were obtained by homologous recombination using an allelic exchange vector, pSS4245. In the first construct, the segment encoding PT subunit S1 was replaced by two mutations (R9K and E129G) that removed PT toxicity and Bp-WWC strain was obtained. In the second construct, a second copy of the whole cluster of PT structural genes containing the above mutations was inserted elsewhere into the chromosome of Bp-WWC and the Bp-WWD strain was obtained. This strain generated increased amounts of $\mathrm{rPT}(3.77 \pm 0.53 \mu \mathrm{g} / \mathrm{mL})$ compared to Bp-WWC (2.61 $\pm 0.16 \mu \mathrm{g} / \mathrm{mL})$ and wild type strain $(2.2 \mu \mathrm{g} / \mathrm{mL})$. In the third construct, a second copy of the prn gene was inserted into the chromosome of Bp-WWD to obtain Bp-WWE. Strain Bp-WWE produced PRN at $4.18 \pm$ $1.02 \mu \mathrm{g} / \mathrm{mL}$ in the cell extract which was about two-fold higher than Bp-WWC $(2.48 \pm 0.10 \mu \mathrm{g} / \mathrm{mL})$ and Bp-WWD $(2.31 \pm$ $0.17 \mu \mathrm{g} / \mathrm{mL}$ ). Purified PTd from Bp-WWD at 0.8-1.6 $\mu \mathrm{g} /$ well did not show any toxicity against Chinese hamster ovary $(\mathrm{CHO})$ cell whereas purified PT from WT demonstrated a cell clustering endpoint at $2.6 \mathrm{pg} / \mathrm{well}$.

Conclusions: We have constructed Bordetella pertussis strains expressing increased amounts of the antigens, rPT or rPT and PRN. Expression of the third antigen, FHA was unchanged (always in excess). These strains will be useful for the manufacture of affordable acellular Pertussis vaccines.

\section{Background}

Pertussis or whooping cough is a severe respiratory disease resulting from colonisation of the upper respiratory tract by the causative organism Bordetella pertussis [1]. Vaccines have been available for decades, comprising

\footnotetext{
* Correspondence: jean.petre@skynet.be; scwpb@mahidol.ac.th 'Department of Biotechnology, Mahidol University, 272 Rama 6 Road, Ratchathewi, Bangkok 10400, Thailand

${ }^{2}$ Bionet-Asia Co. Ltd., Hi-Tech Industrial Estate, 81 Moo 1, Baan-Lane, Bang Pa-In, Ayutthaya 13160, Thailand

Full list of author information is available at the end of the article
}

killed whole cells of $B$. pertussis that are chemically detoxified and formulated with Diphtheria and Tetanus antigens. They are administered as a trivalent DiphtheriaTetanus-Pertussis combination, or in newer combinations with $\mathrm{HBV}$ and Hib, providing additional immunity against Hepatitis B and Haemophilus influenzae type b invasive disease, respectively [2]. The use of whole-cell Pertussis vaccines has been reduced, discouraged, or even banned in a few countries, due to the whole-cell vaccine's questionable safety profile, resulting from high level of
C Biomed Central

C 2012 Buasri et al; licensee BioMed Central Ltd. This is an Open Access article distributed under the terms of the Creative Commons Attribution License (http://creativecommons.org/licenses/by/2.0), which permits unrestricted use, distribution, and reproduction in any medium, provided the original work is properly cited. 
endotoxin and other bacterial toxins associated with killed whole cells [3,4].

Acellular Pertussis vaccines (so-called because they do not contain whole cells but only partially- or extensivelypurified bacterial antigens), were introduced in Japan in 1981 [5]. The higher purity of the component antigens in acellular Pertussis vaccines provided an improved clinical safety profile. These vaccines were introduced in the mid $90 \mathrm{~s}$ in other industrialized countries after extensive clinical trials that demonstrated their safety and efficacy [6]. A broader introduction by the WHO into the Expanded Program of Immunization was, however, hampered by the significantly higher cost of acellular Pertussis vaccines.

A major virulence factor of $B$. pertussis is Pertussis Toxin (PT) $[7,8]$ and pertussis toxoid (PTd) is still the principal antigen in acellular vaccines [8]. Unlike Diphtheria and Tetanus toxins (that can be inactivated by simple treatment with formaldehyde), PT proved more difficult to be inactivated by chemical means [9]. At present, different inactivation processes are in use for commercial manufacture of acellular Pertussis vaccines. Unfortunately, all of them cause extensive denaturation of PT by their chemical treatments.

Two candidate vaccines have been tested using a genetically-inactivated toxin (rPT) [10-12] and one of these candidates was included in a field efficacy trial $[11,12]$. This vaccine was obtained by introducing two mutations into the catalytic subunit S1 of PT, causing abolition of the enzymatic activity of $\mathrm{S} 1$ and thus providing complete absence of toxicity of native PT. This vaccine was formulated with $5 \mu \mathrm{g} r \mathrm{rT}, 2.5 \mu \mathrm{g}$ FHA and $2.5 \mu \mathrm{g}$ PRN and was compared with another vaccine manufactured using classical chemical inactivation, comprising $25 \mu \mathrm{g}$ PTd, $25 \mu \mathrm{g}$ FHA and $8 \mu \mathrm{g}$ PRN. The two vaccines had identical safety and efficacy results in this trial [13]. It was understood that the efficacy obtained with a lower dose of rPT and the other antigens was a result of using native antigens that included native FHA and PRN as the latter also required chemical treatment to inactivate residual traces of toxin when the antigens were derived from wild type B. pertussis.

Unfortunately, the vaccine described above, containing $\mathrm{rPT}$, is not currently available due to unresolved intellectual property issues at the time of planned commercial introduction. Nevertheless, it is clear that the geneticallyengineered approach to detoxification of Pertussis vaccine antigens is an essential element for the design of affordable acellular Pertussis vaccines, as intellectual property rights are expiring.

The vaccines referred to above contained three purified antigens derived from B. pertussis cultures: PTd or rPT, FHA and PRN. PT and even more so PRN are limiting antigens in $B$. pertussis cultures, while FHA is naturally overproduced. Alternative expression systems exist for increasing level of limiting $B$. pertussis vaccine antigens. PRN was expressed in high yield from recombinant Escherichia coli or from the recombinant yeast, Pichia pastoris $[14,15]$. PT subunits were expressed in E. coli, but unfortunately these failed to assemble into the mature toxin and were insufficiently immunogenic to be considered as potential vaccine candidates [16]. It is now understood that assembly and secretion of the mature toxin requires several auxiliary genes that were discovered more recently, and these genes are part of the $p t l$ section of the $p t x-p t l$ operon [17].

In this publication, we report the construction of recombinant $B$. pertussis strains expressing increased levels of rPT or rPT and PRN. These strains were generated by a multiple allelic- exchange process: insertion of the mutations that abolish the catalytic activity of subunit $\mathrm{S} 1$, insertion of a second copy of the $p t x$ cluster of the five PT structural genes of the $p t x-p t l$ operon with their promoter and terminator into an abandoned gene elsewhere on the chromosome, then insertion of a second copy of the prn gene into a second inactive gene locus. The organization of $p t l$ auxiliary genes present in the $p t x-p t l$ operon was not modified. Enhanced production of $\mathrm{rPT}$ and PRN by manipulation of gene copy number has been largely used with multi-copy plasmid vectors and reported to enhance the production of bacterial toxins $[18,19]$, in particular PT [20]. However, genes tandemly repeated in this way may have significantly negative consequences on strain genetic stability in a GMP-regulated, vaccine-manufacturing environment. In addition, PRN expression could also be increased by manipulation of the PRN promoter [21].

The allelic-exchange vectors used in earlier $B$. pertussis recombinant strains require mutations on the chromosome, particularly the mutation affecting $r p s L$ that results from selection of spontaneous streptomycin-resistant mutants as required in earlier allelic-exchange procedures [22]. Such mutations affecting housekeeping genes may impair virulence, hence the expression of virulence factors including PT, FHA and PRN. In contrary, pSS4245 used in this study harbours streptomycin resistant gene from Tn5 which is functional in B. pertussis but not in $E$. coli, hence streptomycin was used to select against $E$. coli donor cell and I-SceI nuclease activity in the plasmid was then functioned as the counter selectable marker in the recombinant $B$. pertussis through subsequent homologous recombination and does not require or leave auxiliary mutations. The strains reported here produce unaltered levels of the other antigens in particular FHA. These constructs will prove useful for the manufacture of affordable human acellular Pertussis vaccines. 


\section{Results}

Mutation of the S1 gene in the B. Pertussis chromosome

To introduce the two mutations R9K and E129G into the S1 subunit, a two-stage approach was used to avoid the possibility of recombination in the region between the two mutations that would cause the loss of one of the mutations. This approach also allowed selection of the desired colonies by simple replica plating on selective media. Firstly, two $E$. coli vectors were constructed in pBluescript II SK + where the wild-type $S 1$ gene was replaced by a chloramphenicol resistance gene $\left(\mathrm{Cm}^{R}\right)$ (Figure 1A) or by a modified $S 1$ gene including the desired mutations (Figure 1B); both flanked by 1.2 and $1.5 \mathrm{~kb}$ of the $S 1$ upstream and downstream regions, respectively. These vectors were then processed and their inserts were introduced into pSS4245. These derivatives were transferred into $E$. coli SM10 for conjugative transfer and allelic exchange into $B$. pertussis strain Tohama. The plasmid pSS5Cm3 generated a replacement of the $S 1$ gene by the $\mathrm{Cm}^{R}$ marker (Figure 2A). The plasmid pSS5S13-9 K-129 $\mathrm{G}$ restored the $S 1$ gene into its original location, now with the two desired mutations (Figure 2B). After selection of isolates on selective media, integration of the $\mathrm{Cm}^{R}$ and modified $S 1$ genes at the expected position was confirmed by PCR amplification (data not shown). The integration of the mutated $S 1$ gene at the designated position was confirmed by PCR with specific primers that could hybridize the upstream 5 and 3 prime downstream flanking regions and internally in the $S 1$ gene (data not shown). The mutations in the $S 1$ gene of the clone selected for further manipulation was confirmed by DNA sequencing. The new strain was designated as Bp-WWC.

\section{Insertion of a second integration site for a second set of PT structural genes}

Initial attempts to increase PT expression by inserting the whole $p t x-p t l$ operon into a multi-copy plasmid compatible with $B$. pertussis failed to deliver useful strains suggesting that the over-expression of PT is potentially toxic and must remain within certain limits to obtain viable strains. In order to increase the PT toxin yield, a second set of PT structural genes was introduced into the Bp-WWC chromosome. To identify an insertion target site, the sequence of the $B$. pertussis Tohama genome (accession number NC_002929) was scanned and many pseudogenes were identified. The DNA sequence (posn. 2905288) between a putative ammonium transporter gene and a putative auto-transporter gene was selected

\section{A. $\mathrm{pSK} 5 \mathrm{Cm} 3$}

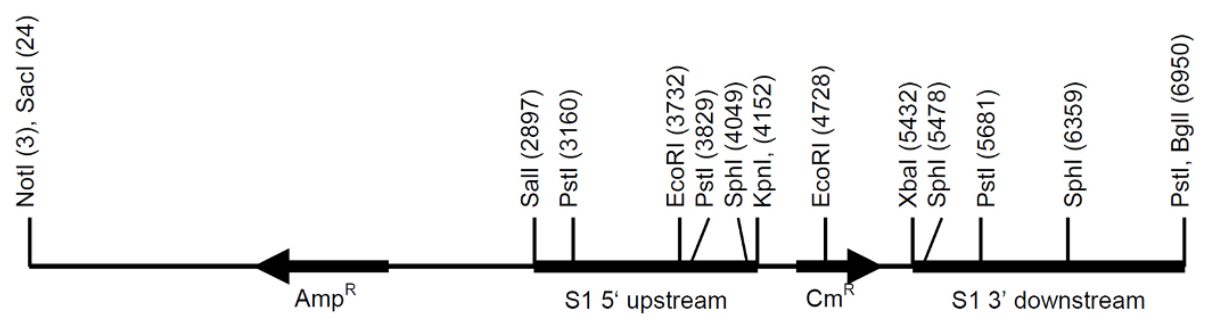

B. pSK5S13-9K-129G

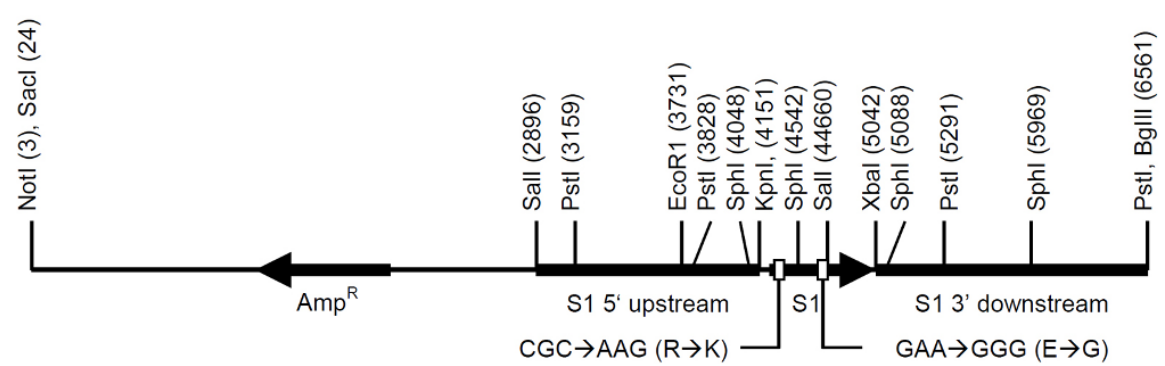

Figure 1 Vectors for the construction of a modified S1 gene into the allelic-exchange vector pSS4245. A: Allelic-exchange element for replacing the S1 gene by a chloramphenicol resistance cassette, inserted between the S1 flanging regions. B: Allelic-exchange element for returning the modified $\mathbf{S 1}$ gene into its exact location in the ptx-pt/ operon. To obtain the allelic exchange, these vectors were linearized and inserted into pSS4245, which was then introduced into B. pertussis by conjugative transfer from E. coli SM10 
A. stage I: replacement of $\mathrm{S} 1$ gene by a $C m^{R}$ gene

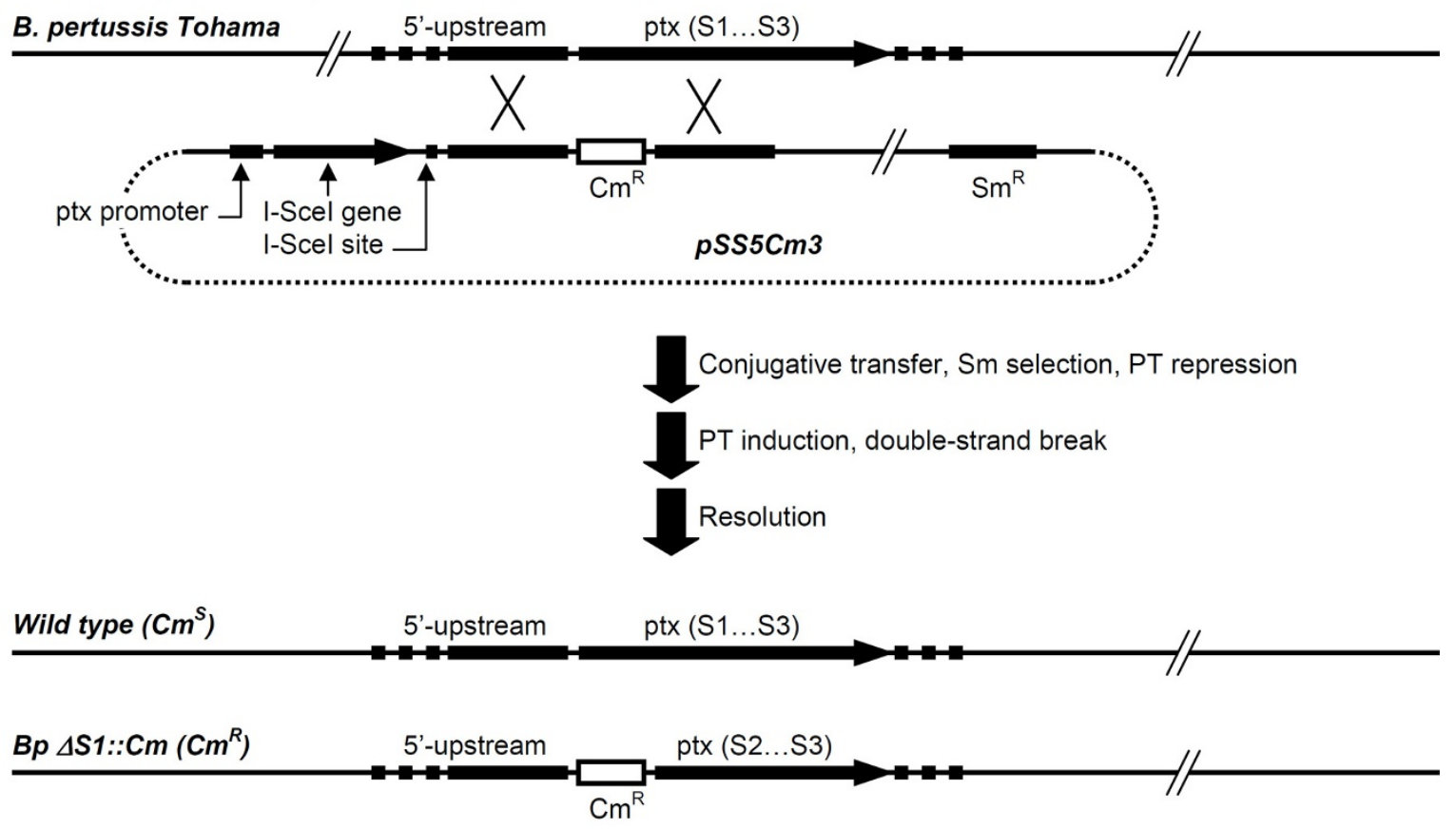

B. Stage II. Replacement of $\mathrm{Cm}^{\mathrm{R}}$ gene by the mutated $\mathrm{S} 1$ gene

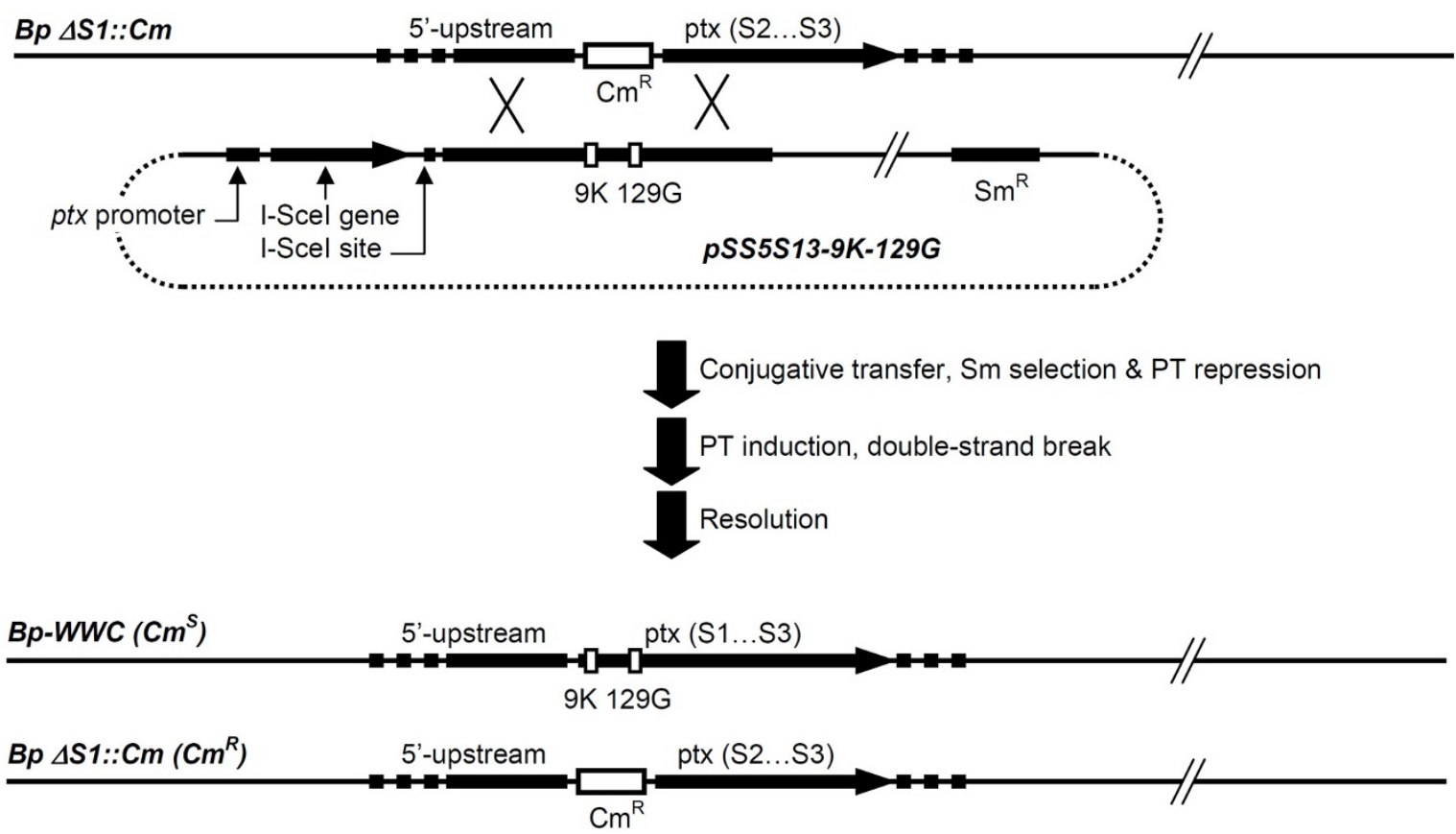

Figure 2 Allelic-exchange procedure. A: Double recombination events leading to the replacement of the S1 gene by a chloramphenicol resistance marker. B: Double recombination events leading to the re-insertion of the modified S1 gene in its original location.

for insertion (posn. 2903988-2905228 and 29052912908277). These genes each carry frameshift mutations which ruin their functionality (Figure $3 \mathrm{~A}$ ). The general strategy outlined in the preceding section was followed.
First, the $E$. coli vector pSKPD5Cm3 was constructed by inserting the $\mathrm{Cm}^{R}$ gene within the regions flanking the selected integration site (Figure 3B). After insertion of the sequences of interest into pSS4245, allelic exchange 


\section{A. Selected insertion site}

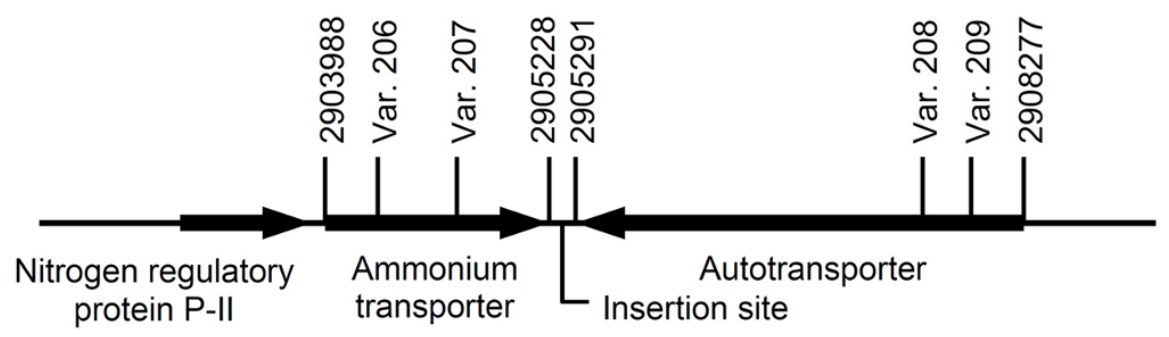

\section{B. pSKPD5Cm3}

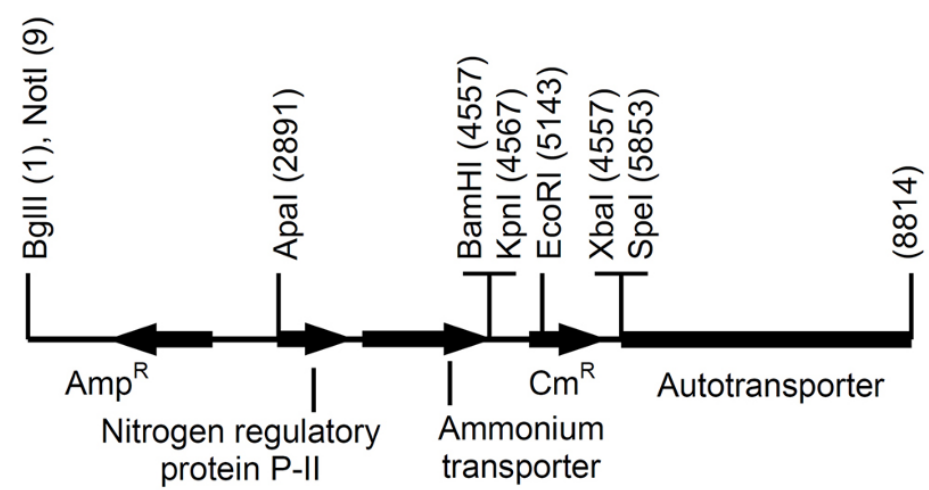

\section{C. pSKptxter with S1* (9K-129G)}

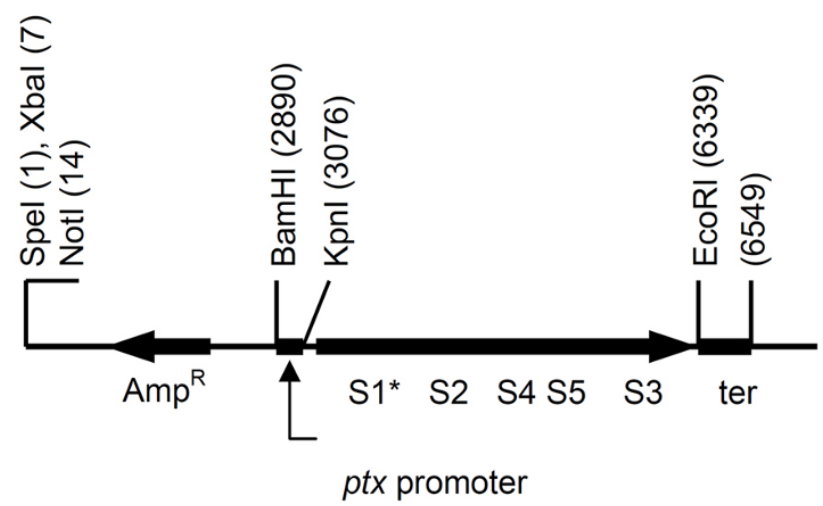

Figure 3 Vectors for the insertion of a second copy of the ptx operon into the B. pertussis chromosome. A: The insertion site for a second copy of the ptx operon was selected between two abandoned genes, each carrying two frameshift mutations. B: Allelic-exchange elements used to insert a chloramphenicol marker into the selected site. C: Schematic structure of the ptx operon with its original promoter. The ptx-ptl terminator was cloned and inserted downstream of the S3 gene. This cluster was finally integrated into the SS4245 derivative to replace the chloramphenicol marker and generate the second allelic-exchange event to insert the second copy of the PT structural genes. 
was selected by the $\mathrm{Cm}^{R}$ marker. Integration of the $\mathrm{Cm}^{R}$ gene at the designated position was confirmed by PCR (data not shown). In the second vector, five PT structural genes with mutated $S 1$ were inserted between the $p t x-p t l$ operon promoter and terminator (following the $S 3$ gene) to generate the vector pSKptxter (Figure 3C). Allelic exchange into the selected target integration inserted a second copy of the functional cluster of the PT structural genes into Bp-WWC strain. The new strain was designated as Bp-WWD. This strain harboured two copies of $p t x$ operon with mutated $S 1$ gene. The result of integration was verified by amplification of the upstream, downstream, and internal regions of the $p t x$ operon, that all showed the expected integration without disruption of the regions where recombination had occurred.

\section{Sequencing of the S1 gene and identification of the R9K and E129G mutations}

Automated sequencing was applied to confirm the presence of the desired mutations. In the case of strain BpWWD that has two integrated copies of the $S 1$ gene, PCR amplification yields, in principle, a mix of the copies of the two genes. An unexpected point mutation in one of the inserts would appear as a double-nucleotide assignment at the corresponding position. The single peak of fluorescence signal at the R9K and E129G positions indicated the correct sequence on Bp-WWC and that of the two copies of $S 1$ in Bp-WWD had identical mutations. The sequence around the two desired mutations is reported in Figure 4 that shows the sequencing records for strain Bp-WWD and the sequence alignments for wild-type Tohama, Bp-WWC and Bp-WWD.

\section{Insertion of a second copy of the prn genes into the Bp- WWD strain}

Due to the low level of PRN expression, a second copy of the prn structural gene (under control of the 246 bp fha promoter and its own terminator) was introduced into the Bp-WWD chromosome (posn. 1345693) between the two pseudogenes of putative exported dehydrogenase (posn. 1344710-1345685) and a putative aspartate racemase (posn. 1345693-1346049) (Figure 5A). The pSKPD2Cm3 E. coli vector was constructed where the $\mathrm{Cm}^{R}$ gene was inserted between the upstream and downstream regions flanking the selected insertion site. Another vector was constructed using the same flanking regions and the prn gene under control of the fha promoter (Figure 5B). After insertion of the $\mathrm{Cm}^{R}$ marker in the desired location, the $\mathrm{Cm}^{R}$ gene was replaced by the prn functional block using the usual allelic-exchange selection and screening procedures.

The $B$. pertussis strains isolated from this construction exercise did not express PRN and the expression level of the other (FHA, PT and hemolysin) antigens was not detectable (data not shown). It was tentatively concluded that the PRN product is toxic if overproduced under control of the stronger fha promoter and only escape mutants having lost the capacity to produce PRN or all virulence factors were viable. It was, therefore, decided to introduce the natural prn promoter in place of the fha promoter. The plasmid pSKPD25FpPRN3 was used to replace the fha promoter by the original prn promoter to generate a functional cassette with its own natural promoter and terminator (Figure $5 \mathrm{C}$ ). This functional cassette was inserted at the selected site by the usual allelic-exchange procedure to obtain a strain with a second non-tandemly-repeated copy of the prn gene under control of its own promoter. The expected insertion was confirmed by PCR amplification with primers binding to the flanking regions internally in the prn gene. This strain was normally viable and was designated as BpWWE.

\section{Genetic stability of PT and PRN constructs in Bp-WWE}

The strain Bp-WWE was cultured and serially sub-cultured in Modified Stainer-Scholte (MSS) medium to reach approximately 50 generations. The last culture was diluted and plated onto MSS agar. Thirty isolated colonies were randomly picked, and analyzed for their $S 1$ and prn genes by PCR (data not shown). The results showed that all colonies contained two copies of $S 1$ and prn genes at the expected positions.

\section{Expression of PT, FHA and PRN in shake flasks}

The production of PT and FHA in shake flask cultures was analyzed by ELISA. Shake flask cultures were all performed in MSS medium containing heptakis(2,6-Odimethyl) $\beta$-cyclodextrin $[23,24]$. At $36 \mathrm{~h}$, the production of PT was about doubled in strain Bp-WWD (3.77 \pm 0.53 $\mu \mathrm{g} / \mathrm{mL})$, compared with Bp-WWC $(2.61 \pm 0.16 \mu \mathrm{g} / \mathrm{mL})$ and wild-type Tohama $(2.2 \mu \mathrm{g} / \mathrm{mL})$ (Table 1$)$, demonstrating that the level of PT expression was a function of the number of copies of the structural gene cluster. FHA in all three recombinant strains was about the same (Table 1). The production of PRN in shake flask cultures of BpWWC, Bp-WWD and Bp-WWE in MSS medium was analyzed by densitometry analysis of Western blot results. PRN amount in the clarified culture supernatants and extract of the separated cells at $60^{\circ} \mathrm{C}$ was assayed. The amount of PRN in cell extract of Bp-WWC and Bp-WWD was similar $(2.48 \pm 0.10$ and $2.31 \pm 0.17 \mu \mathrm{g} / \mathrm{mL}$, respectively). A two-fold increase was found in Bp-WWE (4.18 \pm $1.02 \mu \mathrm{g} / \mathrm{mL}$ ), again showing a good correlation of the level of prn expression to the gene copy number. In all three recombinant strains, the fraction of PRN found in the supernatant fraction in these flask cultures was small or negligible (less than $0.1 \mu \mathrm{g} / \mathrm{mL}$, data not shown). 


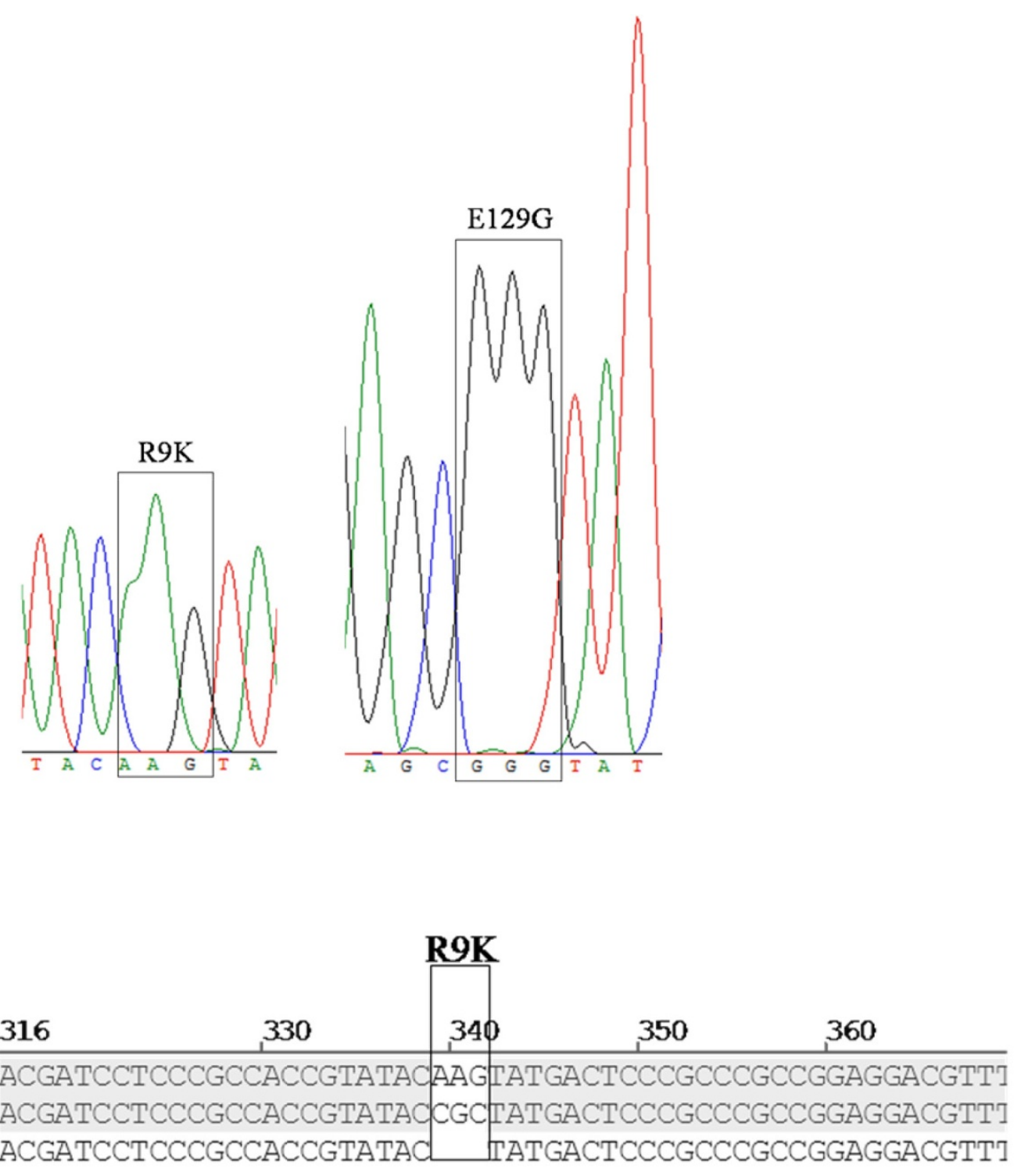

(316) 316

ww $\mathbf{3 1 6 )}$ ACGATCCTCCCGCCACCGTATACAAGPATGACTCCCGCCCGCCGGAGGACGTTI

wid type(159) ACGATCCTCCCGCCACCGTATACCGCFATGACTCCCGCCCGCCGGAGGACGTTI

Consensus(316) ACGATCCTCCCGCCACCGTATAC TATGACTCCCGCCCGCCGGAGGACGTTI

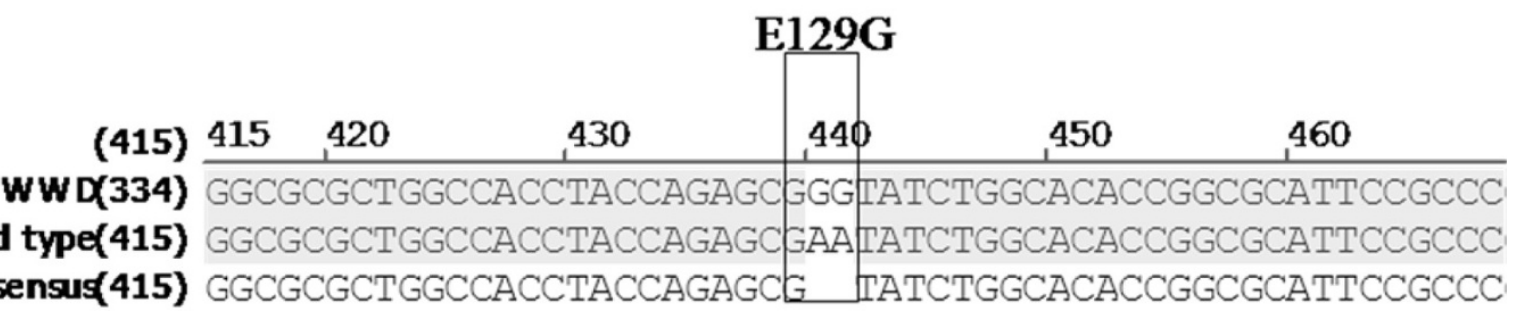

Figure 4 Identification of the R9K and E129G mutations in Bp-WWC and Bp-WWD. Raw sequence data around the mutations are shown for strain Bp-WWD that has two copies of the PT structural cluster. The corresponding sequence alignments are shown for B. pertussis Tohama (consensus sequence) and derivatives Bp-WWC and Bp-WWD. 


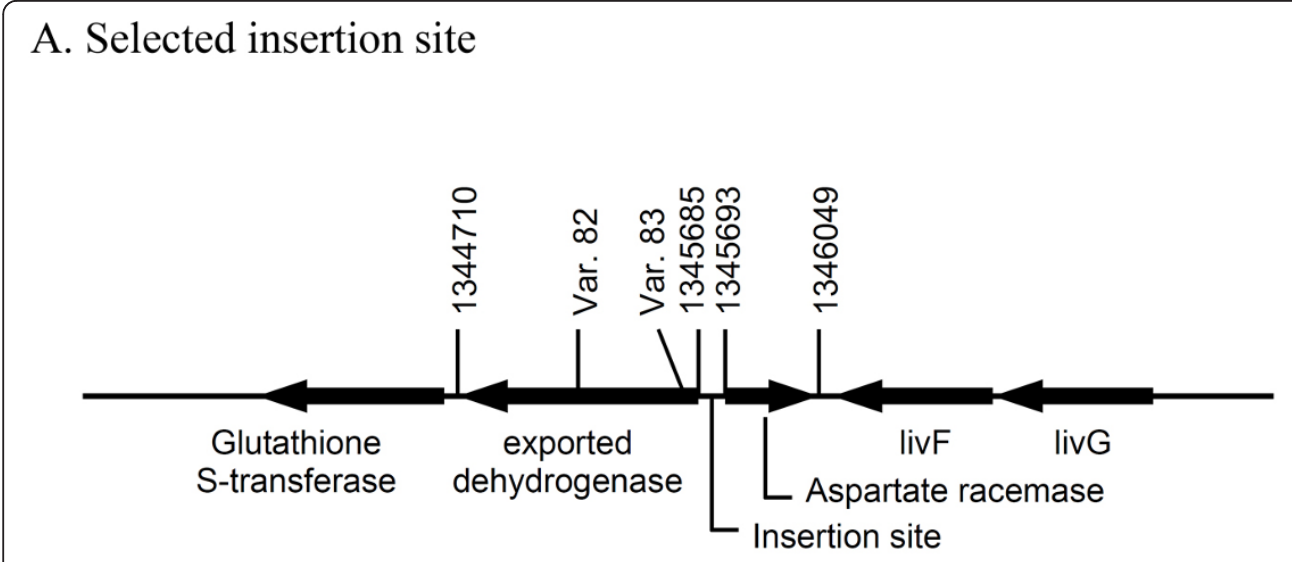

\section{B. pSKPD25FpPRN3}

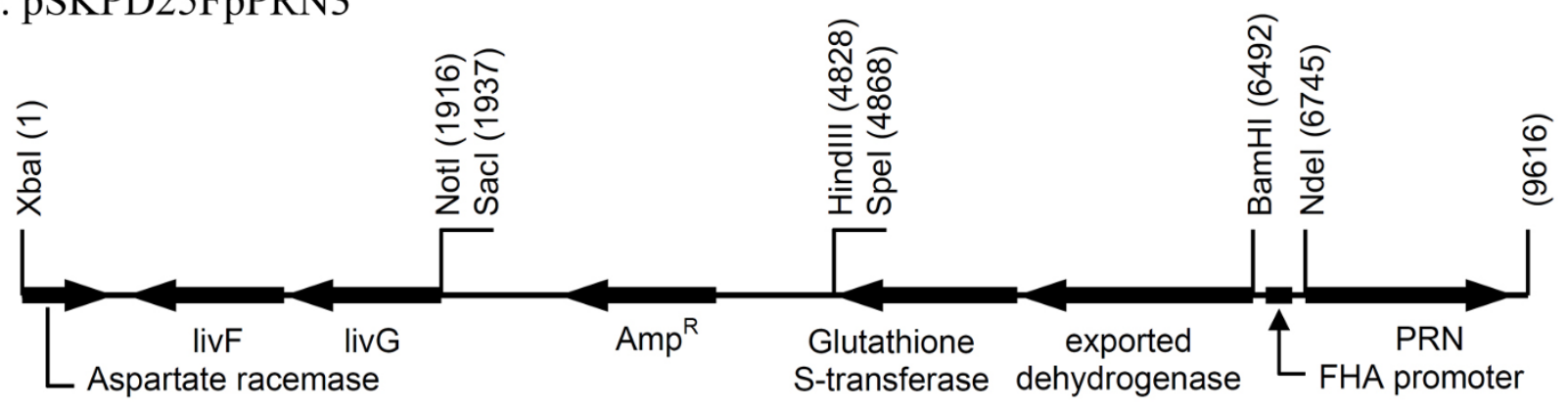

\section{C. pSKPD25PRN3}

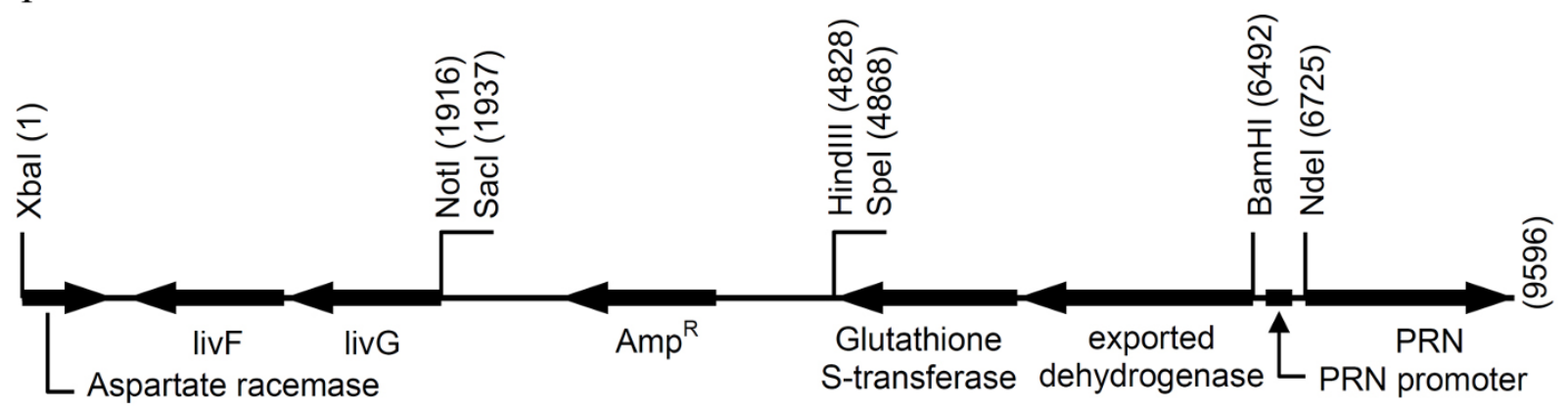

livF : high-affinity branched-chain amino acid transport, ATP-binding protein

livG : leucine/isoleucine/valine transporter ATP-binding subunit

Figure $\mathbf{5}$ Vectors for the insertion of a second copy of the prn gene into the B. pertussis chromosome. A: The insertion site for a second copy of the prn gene was selected between two abandoned genes carrying frameshift mutations and a deletion. B: Schematic structure of the prn gene under control of fha promoter and flanking with target integration site. C: Schematic structure of the prn gene under control of its own promoter and flanking with target integration site.

\section{Assessment of PT inactivation}

PT was purified from culture supernatants using a modification of the process published by Ozcengiz [25] where the initial ammonium sulphate precipitation was replaced by ligand exchange chromatography $[26,27]$. The toxicity of the PT toxin from wild type $B$. pertussis and Bp-WWC (genetically inactivated PT) was analysed and compared by the Chinese hamster ovary $(\mathrm{CHO})$ cell clustering assay [28]. This assay has a much higher sensitivity than other functional assays reported for PT. The native toxin purified from strain $B$. pertussis Tohama demonstrated a clustering endpoint at $2.6 \mathrm{pg}$ 
Table 1 PT, FHA and PRN production by strains Bp-WWC and Bp-WWD and Bp-WWE

\begin{tabular}{lccc}
\hline Strain & PT $(\boldsymbol{\mu g} / \mathbf{m L})$ & FHA $(\boldsymbol{\mu g} / \mathbf{m L})$ & PRN $(\boldsymbol{\mu g} / \mathbf{m L})^{* *}$ \\
\hline Tohama wt & 2.2 & ND $^{*}$ & ND $^{*}$ \\
\hline Bp-WWC & $2.61 \pm 0.16$ & $17.75 \pm 3.30$ & $2.48 \pm 0.10$ \\
\hline Bp-WWD & $3.77 \pm 0.53$ & $14.33 \pm 0.50$ & $2.31 \pm 0.17$ \\
\hline Bp-WWE & $4.49 \pm 0.83$ & $17.08 \pm 2.21$ & $4.18 \pm 1.02$
\end{tabular}

${ }^{*} \mathrm{ND}=$ Not determined ${ }^{* *}$ The amount in cell extract

The values were the mean of 3 independent experiments with standard deviation except the data for PT of Tohama WT was obtained from two independent experiments

per well. The genetically-inactivated PT did not promote clustering at the highest concentrations of 0.8-1.6 $\mu \mathrm{g}$ per sample obtained in this test (Figure 6). This assay can, therefore, detect toxicity reduction by a factor of $5 \times$ $10^{5}$ to $1 \times 10^{6}$, despite limitations imposed by the low solubility of PT. This result demonstrated that PT toxin purified from Bp-WWC was successfully inactivated by insertion of five nucleotide replacements resulting in two amino acid replacements in the PT subunit S1.

\section{Discussion}

Unmarked gene insertion and replacement were successful, using pSS4245 as vector in B. pertussis. After a second homologous recombination to excise the plasmid, no antibiotic gene marker nor any scars were left in the chromosome when compared with the cre-lox system [29] or earlier allelic-exchange procedures used in Bordetella [22]. Overproduction of genetically-deactivated PT toxin was reported in 1992 [20] by using tandem repeats of $p t x$ genes or another copy inserted into the fha gene. The resulting recombinant $B$. pertussis strain overproduced PT up to $80 \mathrm{mg} / \mathrm{L}$. Tandemly-repeated genes are a known potential cause of genetic instability. For this reason, the genome sequence of $B$. pertussis was scanned to look for suitable integration sites. The DNA position between two terminators of pseudo-genes (putative ammonium transporter and putative auto-transporter genes) was selected as integration sites for the $p t x$ cluster. The copy number for the PT structural cluster was limited to two, as overproduction of these virulence factors places a burden on cell metabolism, resulting in slower growth and potentially genetic instability, as shown by preliminary results.

Over-expression of prn gene by the fha promoter to drive higher expression was apparently toxic to growth of $B$. pertussis, possibly in resulting from higher PT expression. Our results showed that replacement of the prn promoter with a stronger one did not provide increased prn expression [21]. Therefore, increasing the gene copy number under the control of the native prn promoter was the approach selected. The fha promoter of the

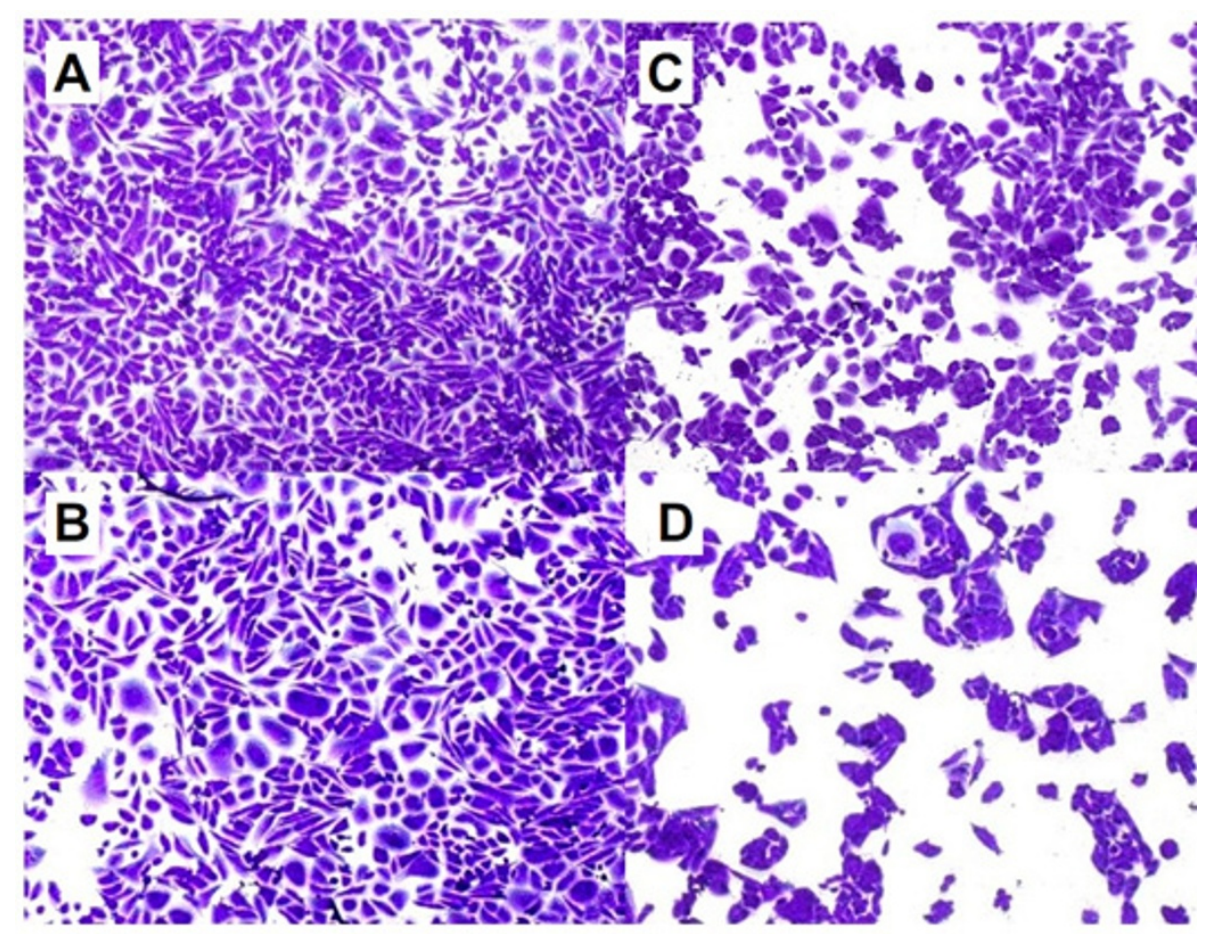

Figure 6 CHO-cell clustering test. The cells were grown to near confluence then dilutions of PT were added and the clustering was scored after 2 days. A: 800 ng PT (strain Bp-WWC). B: Control, no PT added. C: 2.6 pg wt PT (strain Tohama) corresponding to the limit of detection. D. 43 pg wt PT (strain Tohama) 
second gene copy was replaced by the native prn promoter to generate a strain with a second copy of the prn gene and its native promoter inserted into another location on the chromosome. The toxicity of PRN to the host cell was also reported in E. coli [30]. The fha promoter was then replaced by the native prn promoter, then the resulting strain exhibited normal growth in shake flasks and expressed twice the amount of PRN. The distribution of PRN between culture supernatant and cell extract was modified - a larger fraction of total PRN was found in the supernatant although in shake flasks, the quantities of PRN spontaneously released into the supernatant were minimal. The presence of either two copies of mutated $P T$ gene alone or together with two copies of prn in WWC, WWD or WWE did not show any genetic instability as evidenced by serial-subculture experiment. All recombinant strains showed the presence of two copies of corresponding genes and corresponding amount of PT and PRN. Hence, homologous recombination among the homologous copies was not so far found in these strains.

Although bacterial growth in shake-flask culture is limited due to rapid $\mathrm{pH}$ rise and intoxication from release of ammonia by metabolism of the glutamate carbon source [31], shake-flask culture provides a useful indication of the strain's potential under optimized fermentor conditions. The construction of stable strains with enhanced expression of PT (Bp-WWD) or of the two limiting antigens PT and PRN (Bp-WWE) was demonstrated. With enhanced production of PT alone, Bp-WWD could not generate sufficient quantities of PRN, therefore in this case, the use of an independent supply of PRN in recombinant $E$. coli or $P$. pastoris would be required. As the expression level of both PT and PRN has been equally increased in strain BpWWE, it would be expected that matching quantities of the two antigens would also be obtained in higher-density cultures, thereby simplifying vaccine manufacturing operations.

\section{Conclusions}

B. pertussis strains that contains genetically-inactivated S1::R9K-E129G subunits of PT were constructed without leaving any markers or scars in their chromosomes. An about two-fold increase in expression of PT toxin was found in shake flasks by integrating the 5 structural genes (ptx with $S 1$ mutated) under the control of the $p t x-$ $p t l$ operon promoter and terminator between two pseudo-genes on the chromosome. The presence of detoxified $\mathrm{PT}$ was confirmed by the $\mathrm{CHO}$ cell clustering assay. In addition, PRN production was increased by integration of a second copy of the prn gene between other pseudo-genes located elsewhere on the chromosome. The strains were found to be genetically stable in shake flask sub-cultures at higher generation numbers than would be required to reach large-scale fermentations (> $1,000 \mathrm{~L}$ ). These recombinant strains, in particular, strain Bp-WWE (where the ratio of expression of PT and PRN antigens matches the composition of commercial Pertussis vaccines), should enable production of affordable acellular Pertussis vaccines. The lower Cost of Goods (CoG) is provided by the lower dose of native antigens required for adequate immunogenicity and the higher productivity the two limiting antigens PT and PRN.

\section{Methods}

\section{Bacterial strains, plasmids and culture conditions}

All chemicals and reagents used in this study were either molecular biology or analytical grade. Chemicals were purchased from Merck (Germany) and Sigma (USA). Bacterial culture media were obtained from Difco (USA) and Merck. Restriction and modifying enzymes were purchased from New England Biolabs (USA).

E. coli DH5 $\alpha$ (Invitrogen, USA) was used as a cloning host. This strain was grown at $37^{\circ} \mathrm{C}$ in Luria Bertani (LB) medium. The E. coli DH5 $\alpha$ transformants were grown in LB medium supplemented with appropriate antibiotics: amplicillin $(50 \mu \mathrm{g} / \mathrm{mL})$ or chloramphenicol $(15 \mu \mathrm{g} / \mathrm{mL})$. E. coli SM10 and pSS4245 were obtained from Dr. Earle S. Stibitz and used as a conjugative donor strain and an allelic exchange vector, respectively. This strain was grown at $37^{\circ} \mathrm{C}$ in $\mathrm{LB}$ medium supplemented with kanamycin $(50 \mu \mathrm{g} / \mathrm{mL})$. The E. coli SM10 transformants were grown in LB medium supplemented with kanamycin (50 $\mu \mathrm{g} / \mathrm{mL})$, amplicillin $(50 \mu \mathrm{g} / \mathrm{mL})$ and neomycin $(10 \mu \mathrm{g} /$ $\mathrm{mL})$. B. pertussis Tohama was obtained from ATCC (BAA-589). B. pertussis strains were grown at $35^{\circ} \mathrm{C}$ on Bordet-Gengou (BG) agar or MSS medium [32]. One liter of the MSS medium contained $10.7 \mathrm{~g}$ of monosodium glutamate, $0.24 \mathrm{~g}$ of L-proline, $2.5 \mathrm{~g}$ of $\mathrm{NaCl}, 0.5 \mathrm{~g}$ of $\mathrm{KH}_{2} \mathrm{PO}_{4}, 0.2 \mathrm{~g}$ of $\mathrm{KCl}, 0.1 \mathrm{~g}$ of $\mathrm{MgCl}_{2} \cdot 6 \mathrm{H}_{2} \mathrm{O}, 0.02 \mathrm{~g}$ of $\mathrm{CaCl}_{2} \cdot 2 \mathrm{H}_{2} \mathrm{O}, 6.1 \mathrm{~g}$ of Tris base, $10 \mathrm{~g}$ of casamino acids $0.01 \mathrm{~g}$ of $\mathrm{FeSO}_{4} \cdot 7 \mathrm{H}_{2} \mathrm{O}, 0.04 \mathrm{~g}$ of L-cysteine, $0.1 \mathrm{~g}$ of glutathione, $0.02 \mathrm{~g}$ of ascorbic acid, $0.004 \mathrm{~g}$ of niacin and $1 \mathrm{~g}$ of dimethyl- $\beta$-cyclodextrin. Plasmid pBluescript II SK + and pACYC184 were obtained from Stratagene (USA) and New England Biolabs (USA), respectively.

\section{Cloning of S1 flanking regions and insertion of a chloramphenicol gene}

The chromosomal DNA of B. pertussis strain Tohama was used as source material. The upstream region of the S1 gene was amplified by PCR using the 5'F-PT-SalI and 5'R-PT-MCS primers. The latter contained KpnI, XbaI, $B g l \mathrm{II}$ and NotI sites. The amplification product was recovered from agarose gel and purified by QIAEX II Extraction kit (Qiagen, Germany). The 1287 bp amplification product was digested with SalI and NotI and cloned into the $E$. coli vector $\mathrm{pSK} \Delta \mathrm{KpnI}$ digested with 
the same enzymes. pSK $\Delta \mathrm{KpnI}$ was a derivative of pBluescript II SK + where the KpnI site was removed by digestion, trimming 3' protruding end by the Klenow enzyme, and re-circularization. The resulting construct was transformed by heat shock into competent cells of $E$. coli DH5 $\alpha$ and designated as pSK5'. The downstream region was likewise obtained by amplification with the 3'F-PT$\mathrm{XbaI}$ and 3'R-PT-BglII primers. The $1531 \mathrm{bp}$ product was digested with $\mathrm{XbaI}$ and $B g l \mathrm{II}$ and the recovered fragment inserted into pSK5' digested with the same enzymes to obtain pSK53.

The $\mathrm{Cm}^{R}$ gene was obtained from plasmid pACYC184. The gene was amplified using the primers $\mathrm{CmF}-\mathrm{KpnI}$ and CmR-XbaI. The 1295 bp PCR product was purified and digested with $K p n \mathrm{I}$ and $\mathrm{XbaI}$ and inserted into pSK53 cut with the same enzymes. The resulting plasmid was designated as pSK5Cm3. This plasmid incorporated the chloramphenicol resistance gene flanked by the 5'-upstream and 3'-downstream regions of the $S 1$ gene (Figure 1A).

\section{Exchange of the $\mathrm{S} 1$ gene by homologous recombination}

To perform the allelic exchange, vector pSS4245 [33] was used. Plasmid pSK5Cm3 was digested with $\mathrm{SacI}$ and $\mathrm{BglII}$ and the recovered fragment ligated into pSS4245 cut with SacI and BamHI. After transformation into E. coli SM10, the resulting plasmid was designated as pSS5Cm3. Fresh cultures of B. pertussis strain Tohama (4 days on MSS-agar with $20 \mathrm{mM}$ nicotinic acid) and of $E$. coli SM10 harbouring the vector (overnight on LB-agar with ampicillin, kanamycin and chloramphenicol) were scraped and mixed onto agar plates containing LB:MSS (1:1) with $20 \mathrm{mM}$ nicotinic acid and $10 \mathrm{mM} \mathrm{MgCl}$. After 3 h-cultivation at $35^{\circ} \mathrm{C}$, the mix was swabbed onto MSS with $20 \mathrm{mM}$ nicotinic acid, $50 \mu \mathrm{g} / \mathrm{mL}$ streptomycin and $5 \mu \mathrm{g} / \mathrm{mL}$ chloramphenicol. Streptomycin and chloramphenicol were used to select against E. coli SM10 (donor cell) and B. pertussis (recipient cell). The swab growth was streaked onto MSS agar with $5 \mu \mathrm{g} / \mathrm{mL}$ chloramphenicol for the second recombination event. The resulting single colonies were tested by replica plating and a few colonies with the $\mathrm{Sm}^{\mathrm{S}}$ and $\mathrm{Cm}^{\mathrm{R}}$ phenotype were retained for further testing (Figure 2A). The integration of $\mathrm{Cm}^{R}$ gene at designed position was confirmed by PCR using the primers that specifically bind to the upstream $5^{\prime}\left(5^{\prime} \mathrm{F}-\right.$ int and 5'RCM-int primers) and 3' (3'FCM-int and 3'Rint primers) downstream flanking regions and internally in the $\mathrm{Cm}^{R}$ gene. From the PCR analysis, it was confirmed that the 5' and 3' flanking regions were present and the $\mathrm{Cm}^{R}$ gene had been inserted at the expected location in place of the $\mathrm{S} 1$ gene. These verifications also confirmed that the allelic-exchange process had not caused any alteration in the $S 1$ flanking regions where recombination had taken place.

\section{Construction of a modified S1 gene}

The $S 1$ gene was cloned by PCR amplification and mutated by site-directed PCR mutagenesis. The primers S1F-PT-KpnI and S1R-PT-XbaI (Table 2) were used to amplify the gene from chromosomal DNA. The purified PCR product was digested with $\mathrm{XbaI}$ and $K p n \mathrm{I}$ and the recovered 908 bp fragment was ligated into pSK53 cut with the same enzymes. After transformation and colony selection, the resulting plasmid was designated as pSK5S13.

Site-directed PCR mutagenesis used the internal F-R9K and R-R9K primers with the sequence mismatch CGC $\rightarrow$ AAG, causing the R9K substitution. The same procedure was applied to generate the second mutation using the internal mismatched primers F-E129G and R-E129G, to generate the sequence GAA $\rightarrow$ GGG, causing the E129G substitution.

The resulting fragment was digested with $\mathrm{Xba \textrm {I }}$ and $\mathrm{Kp} n \mathrm{I}$ and inserted into pSK53 cut with the same enzymes to obtain plasmid pSK5S13-9 K-129 G (Figure 1B). This was digested with $\mathrm{SacI}$ and $B g l \mathrm{II}$ and the recovered fragment was ligated into pSS4245 cut with SacI and BamHI. After transformation into $E$. coli SM10, the resulting plasmid was designated as pSS5S13-9 K-129 G.

Allelic exchange to insert the modified $S 1$ gene back into its original location in the $B$. pertussis chromosome was performed as above but without selection of the exconjugants by chloramphenicol. The desired strains in this case have lost this marker and therefore screening by replica plating was necessary to identify colonies with the desired phenotype $\mathrm{Cm}^{\mathrm{S}}$ and $\mathrm{Sm}^{\mathrm{S}}$. The resulting Tohama derivative was designated as Bp-WWC (Figure 2B). The integration of the $S 1$ mutated gene at the designated position was confirmed by PCR with the specific primers. The primers could bind the upstream 5' (5'F-int and R-R9K primers), 3' (F-E129G and 3'R-int primers) downstream flanking regions, and inside the $S 1$ gene.

\section{Insertion of a second set of the $\mathbf{5}$ PT structural genes}

The sequences flanking the targeted insertion site (Figure $3 \mathrm{~A})$ were first cloned to obtain pSKPD5Cm3. The upstream $1688 \mathrm{bp}$ fragment was amplified with the primers 5'F-PD-ApaI and 5'R-PD-MCS, digested with ApaI and $K p n \mathrm{I}$, and ligated into $\mathrm{pSK} 5 \mathrm{Cm} 3$ cut with the same enzymes to yield pSKPD5'-Cm. The downstream 2980 bp fragment was amplified with the primers 3'F-PD-MCS and 3'R-PD-BglII, digested with $\mathrm{XbaI}$ and $B g l \mathrm{II}$, and ligated into pSKPD5'-Cm cut with the same enzymes. The resulting plasmid was designated as pSKPD5Cm3 (Figure 3B).

The conjugative construct was obtained by digesting this plasmid with NotI and BglII and ligation into pSS4245 which was digested with NotI and BamHI, resulting in plasmid pSSPD53-Cm. Conjugative transfer and selection for $\mathrm{Sm}^{\mathrm{S}}$ and $\mathrm{Cm}^{\mathrm{R}}$ provided the desired B. pertussis 
Table 2 Primers used for construction

\begin{tabular}{|c|c|}
\hline Name & Sequence \\
\hline$\overline{5^{\prime} F-P T-S a l l}$ & GCGGTCGACGGCGCGCAATGCGGCGCGGAC \\
\hline 5'R-PT-MCS & GGGGGCGGCCGCGAGATCTCTCTAGACGGTACCATCGCGCGACTITGCGCCGAAGGA \\
\hline 3'F-PT-Xbal & CGTTCTAGACCTGGCCCAGCCCCGCCCAAC \\
\hline 3'R-PT-Bgl|I & GGCAGATCTGCAGTTCGAGCAGATCGCCGG \\
\hline CmF-Kpnl & CGCGGTACCTGATGTCCGGCGGTGCTITTG \\
\hline CmR-Xbal & AATCTAGATATCGTCAATTATTACCTCCAC \\
\hline S1F-PT-Kpnl & GATGGTACCGGTCACCGTCCGGACCGTGCT \\
\hline S1R-PT-Xbal & CAGGTCTAGAACGAATACGCGATGCTTTCG \\
\hline R-R9K & GGGCGGGAGTCATACTTGTATACGGTGGCGG \\
\hline F-R9K & CCGCCACCGTATACAAGTATGACTCCCGCCC \\
\hline F-E129G & CCACCTACCAGAGCGGGTATCTGGCACACCGG \\
\hline R-E129G & CCGGTGTGCCAGATACCCGCTCTGGTAGGTGG \\
\hline 5'F-PD-Apal & GGAGGGCCCATGAAACTCGTCATCGCCATCATCAAGCCC \\
\hline 5'R-PD-MCS & TACGGTACCGGATCCCGCATCGCAACAACGGGGTCATCGCGACCC \\
\hline 3'F-PD-MCS & CGTTCTAGAACTAGTCCGCTACCAGGTGTAGCGATAGCCCAGGTG \\
\hline 3'R-PD-BgllI & TGTAGATCTCGGCGAGATACTTGCGTTTCGGCGTTGTCG \\
\hline PtxF-BamHI & TTGGGATCCCAGCGCAGCCCTCCAACGCGCCATCC \\
\hline PtxR-MCS & TCTACTAGTAAGAATTCTCGCGGTATCCGTCAAGGAAAAACATGGAC \\
\hline TerF-EcoRl & GCGGAATTCCGCCTGCCGCCTGCACGCAT \\
\hline TerR-Spel & TCCACTAGTCAAGGGCATCGGGCGCCGGC \\
\hline 5'F-PD2-Spel & CGCACTAGTCTATTCCAGCGGCGGGTCGAAATGGC \\
\hline 5'R-PD2-MCS & CCCCAGGCGGCCGCTGTCTAGAGTGGATCCCAGGCCGATGCGTCCGCCGTGCAGGC \\
\hline 3'F-PD2-Xbal & ATCTCTAGAATGGGCACCTCGGCCACGCTGGCGCTG \\
\hline 3'R-PD2-Notl & AAGTATGCGGCCGCATGAGCGAAACCCTGTTGAAAGTATC \\
\hline CmF-BamHI & CGCGGATCCTGATGTCCGGCGGTGCTTITG \\
\hline FHAproF-BamHI & TCTGGATCCCTGCGCTGGCACCCGCGGCGGGCCG \\
\hline FHAR-MCS & GCCTCTAGATTCATATGATTCCGACCAGCGAAGTGAAGTAAT \\
\hline PRNF-Ndel & CTGGTCGGCATATGAACATGTCTCTGTCACGCATTG \\
\hline PRNF & ATGAACATGTCTCTGTCACGCATTGTCAAGG \\
\hline PRNR-Xbal & GCCTCTAGAGCCTGGAGACTGGCACCGGCCAAGC \\
\hline PrnProF-BamHI & CGGGGATCCGCACCCTGGCCTGCGGGGCGGGACC \\
\hline PRNProR-Ndel & AGACATGTTCATATGGATGCCAGGTGGAGAGCAGA \\
\hline$\overline{5^{\prime} \text { F-int }}$ & CTAGCGTTCGCATACCAAATCCTTGC \\
\hline 5'RCM-int & CCGTAATATCCAGCTGAACGGTCTGG \\
\hline 3'FCM-int & TCTGTGATGGCTTCCATGTCGGCAG \\
\hline $3^{\prime}$ R-int & AGCATGTTGCGGTGTTCCCGGAATG \\
\hline 5'FPD-int & ATGACGGAAAGCCGCATGGGCATTGGGTCC \\
\hline 3'RPD-int & TTCGTACGTGTTCAGGTGCCGATTGCCGG \\
\hline 5'FPD2-int & TGGGCTGGCTGTTCTGGCACGAAACG \\
\hline 3'RPD2-int & TTCATCGAATCGGCGCTGATCCTGGC \\
\hline PRNF-int & AGGTGCAGCCATACATCAAGGCCAGC \\
\hline
\end{tabular}

Sequences with bold letters are the recognition sites for various restriction enzymes used for each construction. Underlined sequences are the sequences of the new codons used for constructing mutant

derivative Bp-PD53-Cm, where the presence of the intact upstream, downstream, and $\mathrm{Cm}^{\mathrm{R}}$ insert was confirmed by PCR amplification. The primers could bind the upstream
5' (5'FPD-int and 5'RCM-int primers), 3' (3'FCM-int and 3'RPD-int primers) downstream flanking regions, and inside the $\mathrm{Cm}^{R}$ gene. 
A functional copy of the ptx operon with its promoter was generated by insertion of the $p t x-p t l$ terminator next to the S3 gene. The five structural genes of PT (modified S1, S2, S4, S5, and S3) with its operon promoter were amplified from Bp-WWC DNA using the primers PtxFBamHI and PtxR-MCS. The 3469 bp amplified product was digested with BamHI and SpeI and the recovered fragment was ligated into pSK $\Delta$ RI cut with the same enzymes to yield pSKptx. Plasmid pSK $\Delta R I$ is a variant of pBluescript II SK + where the EcoRI site has been removed by digestion and filled-in with the Klenow enzyme and re-circularized.

The $p t x$-ptl operon terminator was then amplified with the TerF-EcoRI and TerR-SpeI primers. The 223 bp product was doubly digested with EcoRI and SpeI and ligated into pSKptx cut with the same enzymes. After transformation and colony selection, the resulting plasmid was designated as pSKptxter (Figure 3C). This plasmid was then doubly digested with BamHI and SpeI and ligated into pSSPD5Cm3 cut with the same enzymes to yield the conjugative vector pSSPDptxter. Allelic exchange into BpPD53Cm was performed as described above with replica screening for $\mathrm{Sm}^{\mathrm{S}}$ and $\mathrm{Cm}^{\mathrm{S}}$ colonies to obtain the strain designated as Bp-WWD. The integration of $S 1$ mutated gene at the designated position was confirmed by PCR with specific primers. The primers could bind the upstream 5' (5'FPD-int and R-R9K primers), 3' (F-E129G and 3'RPD-int primers) downstream flanking regions, and internal $S 1$ gene.

\section{Insertion of a second copy of the prn structural gene Integration of a chloramphenicol resistance gene into the target site selected for integrating a second copy of the PRN structural gene}

A derivative of pBluescript SK + lacking the BamHI site was constructed by digestion with the enzyme, filling-in with the Klenow enzyme, and ligation. The resulting plasmid was transformed into $E$. coli and designated as $\mathrm{pSK} \Delta \mathrm{H} 1$.

The sequence of the $B$. pertussis Tohama strain was scanned and pseudo-genes were identified. The DNA sequence (posn. 1345693) between a putative exported dehydrogenase (posn. 1344710-1345685) and a putative aspartate racemase pseudo-gene (posn. 1345693-1346049) was selected as the insertion site. These two genes carried frameshift mutations and were not functional (Figure 5A). The 5'-upstream region to the targeted insertion site was amplified using primers carrying SpeI (5'F-PD2-SpeI) and a multilinker including BamHI and NotI (5'R-PD2-MCS) restriction sites. The amplified product was isolated by gel electrophoresis and doubly digested with SpeI and NotI. The resulting fragment was ligated into a fragment of $\mathrm{pSK} \Delta \mathrm{H} 1$ which was digested with the same enzymes. The resulting plasmid was transformed into $E$. coli and designated as pSKPD25. The 3'-downstream fragment was similarly amplified with primers carrying XbaI(3'F-PD2XbaI) and NotI (3'R-PD2-NotI) restriction sites. After digestion with the same enzymes, the resulting fragment was ligated into a fragment of pSKPD25 digested with the same enzymes. The resulting plasmid was transformed into $E$. coli and designated as PSKPD253.

The chloramphenicol resistance gene was obtained by PCR amplification from plasmid pACYC184 using primers carrying a $\mathrm{BamHI}(\mathrm{CmF}-\mathrm{BamHI})$ and $\mathrm{XbaI}(\mathrm{CmR}-$ $\mathrm{XbaI})$ restriction site. The PCR product was digested with the two enzymes and cloned into pSKPD253 cut with the same enzymes. After ligation, the resulting plasmid was transformed into $E$. coli, verified by restriction analysis and designated as pSKPD25Cm3. The plasmid was digested with NotI and SpeI and the resulting fragment was ligated into pSS4245 which was doubly digested with the same enzymes. The resulting plasmid was designated as pSSP2D5Cm3 and transformed into E. coli SM10. Conjugation was conducted as described above by using Bp-WWD as the recipient $B$. pertussis strain with selection of $\mathrm{Cm}^{\mathrm{R}}$ and $\mathrm{Sm}^{\mathrm{S}}$ single colonies. The integration of $\mathrm{Cm}^{R}$ gene at its designated position was confirmed by PCR with the primers that specifically bind to only the upstream 5' (5'FPD2-int and 5'RCM-int primers), 3' (3'FCM-int and 3'RPD2-int primers) downstream flanking regions, and inside the $\mathrm{Cm}^{R}$ gene.

\section{Integration of prn gene under control of fha promoter}

The structural gene of PRN was amplified from B. pertussis DNA using a primer starting at the ATG start codon (F) and a primer carrying an XbaI (R) restriction site. The 2,808 bp amplified product containing only the coding region and the terminator was treated by an 'A' tailing protocol (Promega, USA). The resulting fragment was cloned into pGEM-T easy vector to obtain a plasmid designated as pGEM-TPRN which was verified by restriction analysis. In an initial workup to create a second copy of the PRN gene driven by the stronger FHA promoter, the FHA promoter was isolated from $B$. pertussis DNA by PCR amplification and inserted ahead of the PRN gene. The FHA promoter was amplified by primers carrying the BamHI (FHAproF-BamHI) and a polylinker containing NdeI-XbaI (FHAR-MCS). The purified product was cut with $\mathrm{BamHI}$ and $\mathrm{XbaI}$ then the recovered DNA fragment was ligated into pSKPD253 cut with the same enzymes. The resulting plasmid designated as pSKPD253Fp was verified by restriction analysis. This plasmid was cut with $N d e \mathrm{I}$ and $\mathrm{XbaI}$, then ligated with the PCR product of the prn gene which was amplified from PGEMTPRN by PRNF-NdeI and PRNR-XbaI primers and cut with the same enzymes. The resulting plasmid was designated as pSKPD25FpPRN3 (Figure 5B). The conjugative construct was obtained by digesting this plasmid with NotI and SpeI and ligation into pSS4245 digested with the same enzymes. 
The resulting plasmid was designated as pSSPD2FpPRN. This construct was inserted at the selected location of the Bp-WWD chromosome to replace the chloramphenicol resistance marker introduced using the usual allelicexchange procedures and screening as described above.

\section{Expression of prn gene under control of prn promoter}

The PRN promoter was cloned by PCR amplification of the $B$. pertussis DNA using primers with the restriction sites BamHI (PrnProF-BamHI) and NdeI (PRNProRNdeI). The plasmid pSKPD25FpPRN3 was cut with BamHI and NdeI to generate a fragment which had lost the FHA promoter. The PRN promoter was ligated in its place. After transformation into E. coli and verification by restriction analysis, the resulting plasmid was designated as pSKPD25PRN3 (Figure 5C). The plasmid was cut with NotI and inserted into pSS4245 cut with the same enzyme. The resulting construct, pSSPD2prn was transferred into E. coli SM10 to conduct the allelic exchange. The resulting B. pertussis strain was designated as Bp-WWE. Integration of the prn gene at its designated position was confirmed by PCR with the primers that specifically bind only to the upstream 5' (5'FPD2-int and PRNProR-NdeI primers), 3' (PRNF-int and 3'RPD2-int primers) downstream flanking regions, and inside the prn gene.

\section{PT, FHA and PRN expression in shake flask culture}

The Bp-WWC, Bp-WWD and Bp-WWE strains were grown in shake flasks with $100 \mathrm{~mL}$ MSS medium supplemented with methylated $\beta$-cyclodextrin $(1 \mathrm{~g} / \mathrm{l})$ at $35^{\circ} \mathrm{C}$ with shaking speed of $200 \mathrm{rpm}$. After 32-48 h of growth, the culture supernatants were collected and assayed by ELISA to quantify the PT and FHA expression level. As PRN releasing from its membrane-bound precursor is the result of an imprecise cleavage by unidentified proteases [34], PRN expression was determined by Western blot with densitometric analysis to evaluate the integrity of the antigen. This assay was conducted both on the clarified culture supernatant and the cell extract obtained by heating cell suspension in isotonic buffer $(10 \mathrm{mM}$ Tris- $\mathrm{HCl}$ $\mathrm{pH} 8.0,150 \mathrm{mM} \mathrm{NaCl}, 0.002 \% \mathrm{NaN}_{3}$, and $1 \mathrm{mM}$ PMSF) at $60^{\circ} \mathrm{C}$ for $30 \mathrm{~min}$ and the supernatant was collected after centrifugation at $10,000 \times \mathrm{g}, 4^{\circ} \mathrm{C}$ for $30 \mathrm{~min}$.

\section{ELISA assay for PT and FHA}

Purified rabbit polyclonal antibodies against PT or FHA (NLAC, Thailand) with the dilution of 1:1000 in carbonate/bicarbonate buffer ( $\mathrm{pH}$ 9.6) were coated in 96-well plates (NUNC Maxisorp, Denmark) for $100 \mu \mathrm{L}$ per well and incubated overnight at $4^{\circ} \mathrm{C}$. After 3 time-washing with phosphate-buffered saline $\mathrm{pH} 7.4$ containing $0.1 \%$ Tween 20 (PBST), blocking was performed using $100 \mu \mathrm{L}$ per well of $3 \%$ bovine serum albumin (BSA)-PBST then incubated at $37^{\circ} \mathrm{C}$ for $1 \mathrm{~h}$. After discarding the blocking buffer and washing, dilutions of the standard PT, FHA or samples were loaded and incubated at $37^{\circ} \mathrm{C}$ for $1 \mathrm{~h}$. Then, anti-PT mouse monoclonal antibody (Abcam, USA) at 1:30,000 dilution or anti-FHA mouse monoclonal antibody (NIBSC, UK) at 1:10,000 dilution in blocking buffer was added and incubated under the same conditions. After washing the wells for three times with PBST, $100 \mu \mathrm{L}$ of rabbit anti-mouse $(\mathrm{H}+\mathrm{L})$ IgG-HRP conjugate (Abcam, USA) in blocking buffer at 1:10,000 dilution was used as secondary antibody and incubated for $37^{\circ} \mathrm{C}$ for $1 \mathrm{~h}$. After washing with PBST, $100 \mu \mathrm{L}$ of enzyme substrate, 3,3',5,5'tetramethylbenzidine (KPL, USA), was added. The colour reaction was terminated with $1 \mathrm{~N} \mathrm{HCl}, 100 \mu \mathrm{L}$ per well. Optical density was measured at $450 \mathrm{~nm}$ using a microtiter plate reader. ELISA assay for PT and FHA of each recombinant strain was done in three replicates using three independent cultures.

\section{Western blot assay for PRN}

Dilutions of standard PRN and samples were resolved in a 10\% SDS-PAGE gel then transferred to a PVDF membrane using a semi-dry blotting system. The membrane was blocked with 5\% skim milk in PBST for $1 \mathrm{~h}$. After discarding the blocking solution, the membrane was incubated with $20 \mathrm{~mL}$ anti-PRN sheep serum (NIBSC, UK) at 1:10,000 dilution in blocking buffer for $1 \mathrm{~h}$, then washed three times with PBST. The membrane was then incubated under the same conditions with $20 \mathrm{~mL}$ of rabbit anti-sheep IgG-HRP conjugate (Santa Cruz Biotechnology, USA) and washed again. The membrane was then immersed in 3,3'-diaminobenzamidine until the brown colour developed. The reaction was terminated by rinsing 2-3 times with de-ionized water, then left to dry at room temperature. Western blot of PRN of the three recombinant strains was performed in three replicates using cell extracts from three independent cultures of each strain. The membranes were scanned and converted to a picture file. PRN concentrations were derived by densitometric analysis of the sample and reference bands using ImageJ software http://rsbweb.nih.gov/ij/.

\section{Genetic stability}

The strains were cultured in $100 \mathrm{~mL}$ MSS medium at $35^{\circ} \mathrm{C}$ and agitated at $200 \mathrm{rpm}$ for $48 \mathrm{~h}$, then $0.1 \mathrm{~mL}$ of culture was transferred into $100 \mathrm{~mL}$ MSS and incubated under the same conditions. This step was repeated four more times. Each transfer corresponded to 50 generations. The culture was diluted and plated on MSS agar. Thirty isolated colonies of a final plating were randomly picked and analysed by PCR to detect the expected presence of $p t x$ and prn inserts.

\section{$\mathrm{CHO}$ cell-clustering assay}

$\mathrm{CHO}$ cell clustering activity was determined by the method of Hewlett et al. [28] In short, $\mathrm{CHO}$ cells were 
cultured in the cRPMI 1640 medium supplemented with $10 \%$ fetal bovine serum. The cells were incubated at $37^{\circ} \mathrm{C}$ under $5 \% \mathrm{CO}_{2}$ atmosphere. After trypsinization, $200 \mu \mathrm{L}$ of $\mathrm{CHO}$ cell suspension at density of $2 \times 10^{4}$ cells/mL were seeded in a 96-well micro-culture plate. Test samples and reference PT toxin were serially diluted at ten-fold intervals in phosphate-buffered saline (PBS) $\mathrm{pH} 7.4$ and a $25 \mu \mathrm{L}$ volume of the dilutions was added to each well. After incubation for $48 \mathrm{~h}$ under the same conditions to permit maximal clustering, cells were stained with crystal violet and photographed.

\section{Abbreviation}

PT: Pertussis toxin; PRN: Pertactin; FHA: Filamentous hemagglutinin; rPT: recombinant Pertussis toxin; ELISA: Enzyme-Linked Immunosorbent Assay

\begin{abstract}
Acknowledgements
We are grateful to Dr. Earle S. Stibitz, at the Division of Bacterial, Parasitic, and Allergenic Products, Center for Biologics Evaluation and Research, Food and Drug Administration, USA, for the generous provision of pSS4245, E. coli SM10 and directions for their use for conjugative transfer and allelic exchange in Bordetella. We thank Dr. Kanchana Kenkoom, at the National Laboratory Animal Center (NLAC), Mahidol University, Thailand and Prof. Watchara Kasinrerk at the Biomedical Technology Research Unit, Chiang Mai University, Thailand, for the preparation of polyclonal and monoclonal antibodies. We acknowledge the participation of Assoc. Prof. Worawidh Wajjwaku, Department of Pathology of Veterinary Medicine, Kasetsart University, Thailand, for performing the PT toxicity tests in CHO cells. We thank Dr. Pramvadee Wongsangchandra of the Department of Biotechnology, Faculty of Science, Mahidol University, and Eiakalak Hemjinda, Greanggrai Hommalai, Kulnaree Phetrong, Nantidaporn Ruangchan, and Chutintorn Suadee of Bionet-Asia Co. Ltd., Hi-Tech Industrial Estate, Bang PaIn, Thailand, for their participation to seeding procedures, purification of antigens and assay development.

\section{Author details}

'Department of Biotechnology, Mahidol University, 272 Rama 6 Road Ratchathewi, Bangkok 10400, Thailand. ${ }^{2}$ Bionet-Asia Co. Ltd., Hi-Tech Industrial Estate, 81 Moo 1, Baan-Lane, Bang Pa-In, Ayutthaya 13160, Thailand. ${ }^{3}$ Mahidol University-Osaka University Collaborative Research Center for Bioscience and Biotechnology (MU-OU:CRC), Faculty of Science, Mahidol University, 272 Rama 6 Road, Ratchathewi, Bangkok 10400, Thailand.
\end{abstract}

\section{Authors' contributions \\ WB, AL and PP conceived the study. WP, CB, Al and JP designed the experiments. WB wrote the draft of manuscript, JP and WP revised the manuscript. All authors read and approved the final version of the manuscript.}

\section{Competing interests}

The authors declare that they have no competing interests.

Received: 29 November 2011 Accepted: 23 April 2012

Published: 23 April 2012

\section{References}

1. Mattoo S, Cherry JD: Molecular pathogenesis, epidemiology, and clinical manifestations of respiratory infections due to Bordetella pertussis and other Bordetella subspecies. Clin Microbiol Rev 2005, 18:326-382.

2. Aristegui J, Usonis V, Coovadia H, Riedemann S, Win KM, Gatchalian S, Bock HL: Facilitating the WHO expanded program of immunization: the clinical profile of a combined diphtheria, tetanus, pertussis, hepatitis B and Haemophilus influenzae type b vaccine. Int J Infect Dis 2003, 7:143-151.
3. Miller DL, Ross EM, Alderslade R, Bellman MH, Rawson NS: Pertussis immunisation and serious acute neurological illness in children. $\mathrm{Br}$ Med J (Clin Res Ed) 1981, 282:1595-1599.

4. Stuart-Harris C: Benefits and risks of immunization against pertussis. Dev Biol Stand 1979, 43:75-83.

5. Sato $Y$, Sato H: Development of acellular pertussis vaccines. Biologicals 1999, 27:61-69.

6. Brown B, Greco D, Mastrantonio P, Salmaso S, Wassilak S: Pertussis vaccine trials. Trial synopses. Dev Biol Stand 1997, 89:37-47.

7. Monack D, Munoz JJ, Peacock MG, Black WJ, Falkow S: Expression of pertussis toxin correlates with pathogenesis in Bordetella species. J Infect Dis 1989, 159:205-210.

8. Weiss AA, Hewlett EL: Virulence factors of Bordetella pertussis. Annu Rev Microbiol 1986, 40:661-686.

9. Munoz JJ, Arai H, Cole RL: Mouse-protecting and histamine-sensitizing activities of pertussigen and fimbrial hemagglutinin from Bordetella pertussis. Infect Immun 1981, 32:243-250.

10. Loosmore SM, Zealey GR, Boux HA, Cockle SA, Radika K, Fahim RE, Zobrist GJ, Yacoob RK, Chong PC, Yao FL, et al: Engineering of genetically detoxified pertussis toxin analogs for development of a recombinant whooping cough vaccine. Infect Immun 1990, 58:3653-3662.

11. Nencioni L, Pizza M, Bugnoli M, De Magistris T, Di Tommaso A, Giovannoni F, Manetti R, Marsili I, Matteucci G, Nucci D, et al: Characterization of genetically inactivated pertussis toxin mutants: candidates for a new vaccine against whooping cough. Infect Immun 1990, 58:1308-1315.

12. Pizza M, Covacci A, Bartoloni A, Perugini M, Nencioni L, De Magistris MT, Villa L, Nucci D, Manetti R, Bugnoli M, et al: Mutants of pertussis toxin suitable for vaccine development. Science 1989, 246:497-500.

13. Greco D, Salmaso S, Mastrantonio P, Giuliano M, Tozzi AE, Anemona A, Ciofi degli MLAtti, Giammanco A, Panei P, Blackwelder WC, et al: A controlled trial of two acellular vaccines and one whole-cell vaccine against pertussis. Progetto Pertosse Working Group. N Engl J Med 1996, 334:341-348.

14. Makoff AJ, Oxer MD, Ballantine SP, Fairweather NF, Charles IG: Protective surface antigen P69 of Bordetella pertussis: its characterization and very high level expression in Escherichia coli. Biotechnology (N Y) 1990, 8:1030-1033.

15. Romanos MA, Clare JJ, Beesley KM, Rayment FB, Ballantine SP, Makoff AJ, Dougan G, Fairweather NF, Charles IG: Recombinant Bordetella pertussis pertactin (P69) from the yeast Pichia pastoris: high-level production and immunological properties. Vaccine 1991, 9:901-906.

16. Nicosia A, Bartoloni A, Perugini M, Rappuoli R: Expression and immunological properties of the five subunits of pertussis toxin. Infect Immun 1987, 55:963-967.

17. Kotob SI, Hausman SZ, Burns DL: Localization of the promoter for the ptl genes of Bordetella pertussis, which encode proteins essential for secretion of pertussis toxin. Infect Immun 1995, 63:3227-3230.

18. Clare JJ, Rayment FB, Ballantine SP, Sreekrishna K, Romanos MA: High-level expression of tetanus toxin fragment $\mathrm{C}$ in Pichia pastoris strains containing multiple tandem integrations of the gene. Biotechnology ( $N$ Y) 1991, 9:455-460.

19. Rappuoli R: Isolation and characterization of Corynebacterium diphtheriae nontandem double lysogens hyperproducing CRM197. Appl Environ Microbiol 1983, 46:560-564.

20. Zealey GR, Loosmore SM, Yacoob RK, Cockle SA, Herbert AB, Miller LD, Mackay NJ, Klein MH: Construction of Bordetella pertussis strains that overproduce genetically inactivated pertussis toxin. Appl Environ Microbiol 1992, 58:208-214.

21. Loosmore SM, Yacoob RK, Zealey GR, Jackson GE, Yang YP, Chong PS, Shortreed JM, Coleman DC, Cunningham JD, Gisonni L, et al: Hybrid genes over-express pertactin from Bordetella pertussis. Vaccine 1995, 13:571-580.

22. Stibitz S: Use of conditionally counterselectable suicide vectors for allelic exchange. Methods Enzymol 1994, 235:458-465.

23. Imaizumi A, Suzuki Y, Ono S, Sato H, Sato Y: Heptakis(2,6-O-dimethyl)betacyclodextrin: a novel growth stimulant for Bordetella pertussis phase I. J Clin Microbiol 1983, 17:781-786.

24. Imaizumi A, Suzuki Y, Ono S, Sato H, Sato Y: Effect of heptakis (2,6-Odimethyl) beta-cyclodextrin on the production of pertussis toxin by Bordetella pertussis. Infect Immun 1983, 41:1138-1143. 
25. Ozcengiz E, Kilinc K, Buyuktanir O, Gunalp A: Rapid purification of pertussis toxin (PT) and filamentous hemagglutinin (FHA) by cation-exchange chromatography. Vaccine 2004, 22:1570-1575.

26. Capiau C, Desmons P: Method for isolating and purifying Bordetella pertussis antigenic factors. In Book Method for isolating and purifying Bordetella pertussis antigenic factors (Editor ed.^eds.), vol. 5391715 City: SmithKline Beecham Biologicals; 1995.

27. Chong P, Jackson G, Cwyk W, Klein M: Simultaneous determination of Bordetella pertussis toxin and filamentous haemagglutinin concentrations by hydroxyapatite high-performance liquid chromatography. $J$ Chromatogr 1990, 512:227-236.

28. Hewlett EL, Sauer KT, Myers GA, Cowell JL, Guerrant RL: Induction of a novel morphological response in Chinese hamster ovary cells by pertussis toxin. Infect Immun 1983, 40:1198-1203.

29. Sauer B: Functional expression of the cre-lox site-specific recombination system in the yeast Saccharomyces cerevisiae. Mol Cell Biol 1987, 7:2087-2096.

30. Charles I, Fairweather N, Pickard D, Beesley J, Anderson R, Dougan G, Roberts M: Expression of the Bordetella pertussis P.69 pertactin adhesin in Escherichia coli: fate of the carboxy-terminal domain. Microbiology 1994, 140(Pt 12):3301-3308.

31. Frohlich BT, De Bernardez Clark ER, Siber GR, Swartz RW: Improved pertussis toxin production by Bordetella pertussis through adjusting the growth medium's ionic composition. J Biotechnol 1995, 39:205-219.

32. Stainer DW, Scholte MJ: A simple chemically defined medium for the production of phase I Bordetella pertussis. J Gen Microbiol 1970, 63:211-220.

33. Inatsuka CS, Xu Q, Vujkovic-Cvijin I, Wong S, Stibitz S, Miller JF, Cotter PA: Pertactin is required for Bordetella species to resist neutrophil-mediated clearance. Infect Immun 2010, 78:2901-2909.

34. Capiau C, Carr SA, Hemling ME, PI ainchamp D, Conrath K, Hauser P, Simoen E, Comberbach M, Roelants P, Desmons $P$, et al: Purification, characterization, and immunological evaluation of the 69-kDa outer membrane protein of Bordetella pertussis Proceedings of the sixth international symposium on pertussis. Bethesda, Md.: Department of Health and Human Services, United States Public Health Service, Food and Drug Administration; 1990, 75-85.

doi:10.1186/1471-2180-12-61

Cite this article as: Buasri et al:: Construction of Bordetella pertussis strains with enhanced production of genetically-inactivated Pertussis Toxin and Pertactin by unmarked allelic exchange. BMC Microbiology 2012 12:61.

\section{Submit your next manuscript to BioMed Central and take full advantage of:}

- Convenient online submission

- Thorough peer review

- No space constraints or color figure charges

- Immediate publication on acceptance

- Inclusion in PubMed, CAS, Scopus and Google Scholar

- Research which is freely available for redistribution

Submit your manuscript at www.biomedcentral.com/submit 